\title{
VORWORT
}

Die vorliegende Jahresgabe, die einen wesentlichen Beitrag zur Winckelmann-Forschung bedeutet, hat uns unser Mitglied Universitätsprofessor Dr. Bernhard Schweitzer vermittelt. Die Verfasserin, Frau Dr. Ingrid Kreuzer, Tübingen, hat uns in liebenswürdiger Weise ihre Arbeit überlassen, und wir danken beiden herzlich für die Förderung der Aufgaben unserer Gesellschaft. Zu großem Dank sind wir auch der Deutschen Akademie der Wissenschaften zu Berlin und der Geschwister Boehringer-Stiftung für Geisteswissenschaften zu Ingelheim a. Rh. verpflichtet, die die Druckkosten übernommen haben.

\section{Arthur Schulz}

Vorsitzender der Winckelmann-Gesellschaft Stendal 